\title{
1 RESEARCH OF TECHNICAL SYSTEMS OF PROCESSES 4 OF MIXING MATERIALS
}

ABSTRACT

The process of a vibration concrete mixer has been investigated. Well-coordinated equations of motion of the system «mixer - concrete mixture» and their solutions have established the regularities of changing the parameters of the mixer at different angles of inclination. An experimental design of vibration-gravitational mixing has been developed and manufactured, and studies of the working process of mixing efficiency have been carried out on it. Research carried out at three warehouses of concrete mixtures confirmed the assumptions accepted in the work, the assumption and the selected physical and mathematical model of the "Mixer - concrete mixture» system. The use of a vibration concrete mixer made it possible to reduce the duration of mixing by 2.5-3 times in comparison with conventional free mixing concrete mixers. Provides the preparation of hard concrete mixtures and improving the quality of concrete mixtures due to the destruction of defective aggregates and a more uniform distribution of the binder throughout the volume of the mixed mixture.

An algorithm and method for calculating the main parameters of a vibration concrete mixer have been developed.

\section{KEYWORDS}

Vibration mixer, material, physical, mathematical model, equation, resonance, parameters, experimental setup, vibration frequency, productivity.

\subsection{JUSTIFICATION OF THE CONSTRUCTIVE DIAGRAM OF THE TECHNICAL SYSTEM FOR THE PROCESSES OF MIXING MATERIALS}

The technical systems for the processes of mixing materials include mixers, which are classified according to the characteristics: the principle of mixing, the nature of work, the method of installation [1, 2]. According to the principle of mixing the components, concrete mixers are divided into two classes: with free mixing of materials (gravitational) and with forced [3]. It is most expedient to use gravity mixers to prepare more plastic mixtures with lower resistivity when mixing them [4]. For the preparation of hard concrete mixes, mixers with forced mixing of materials are most effective. At the same time, modern requirements for the quality of concrete mixtures require the use of more efficient mixing equipment. Such equipment includes mixers using vibration effect [5]. In such a mixer, up to five component movements take place, and mixing is carried out simultaneously 
by translational and rotational movements of the components of the mixture. A free-mixing vibration concrete mixer (Fig. 4.1) includes a cone-cylindrical mixing drum 1, which is mounted on a base plate 3 with the help of elastic shock absorbers 2.

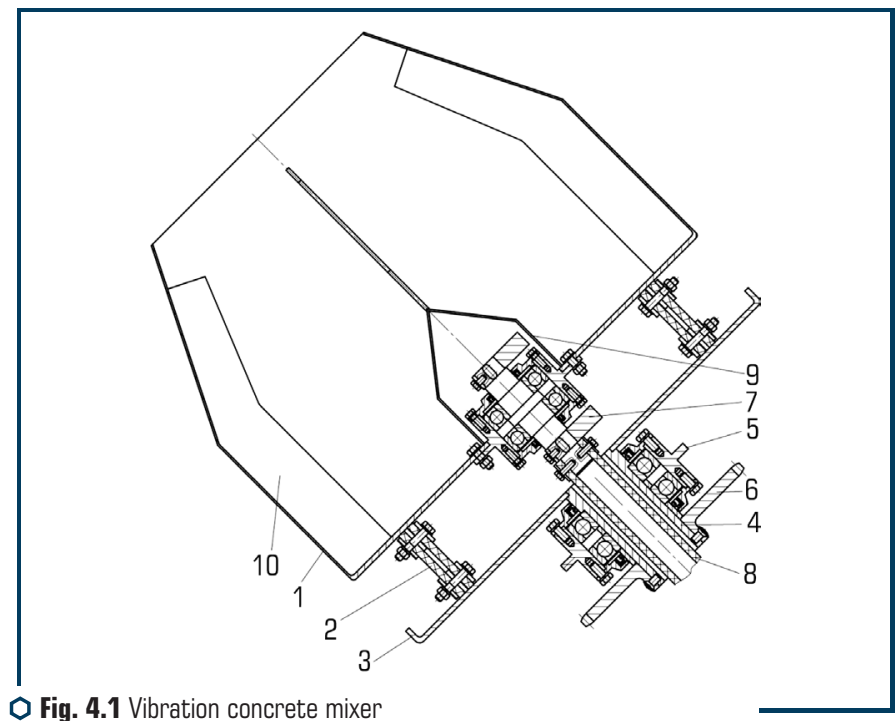

This base plate 3 is rigidly connected in its central part to a hollow drive shaft 4, mounted in a bearing support 5 . At the end of the drive shaft 4 , a sprocket 6 is mounted, connected to an electromechanical drive (not shown). On the bottom of the mixing drum 1, a circular vibration exciter 7 is mounted, which is half mounted in the inner cavity of the mixing drum. In this case, the vibration exciter of circular vibrations 7 is connected to the drive motor (not shown in the Fig. 4.1) using a flexible shaft 8 passing inside the hollow drive shaft 4 . Inside the mixing drum, the vibration exciter of circular vibrations 7 is closed with a cone-cylinder protective cap. drum. Blades 10 are located inside the mixing drum 1 . The bearing support 5 is mounted on the traverse of the fixing and tipping mechanism.

Free mixing vibration concrete mixer works as follows.

Mixing drum 1 is installed at an angle of $40-45$ degrees to the horizon and is loaded with a portioned amount of sand, crushed stone and cement. Pour in the required amount of water. The mixer drum drive is included. In this case, the rotating mixing drum using the blades 10 provides mechanical mixing of the mixture. This preliminary mixing of the components of the mixture continues for 15-20 s.

Then, with the help of the fixing and tipping mechanism, the mixing drum is set in a vertical position and the vibration exciter of circular vibrations 7. Under the influence of vibration, 
the mixing drum performs complex vibration movements that cause variable amplitude-frequency deformations in the concrete mixture. The vibration action of the applied simultaneous torsional and circular vibrations ensures efficient mixing of the concrete mixture. The duration of vibromechanical mixing, vibration treatment and vibration activation is 15-25 s, depending on the concrete mix. After the end of vibromechanical mixing, the vibration exciter of vibrations is turned off and, using the fixing and overturning mechanism, the mixing drum is transferred to its original position. The drum is installed at an angle of $40-45$ degrees to the horizon and the process of mixing the mixture continues for 10-12 seconds until a homogeneous state is obtained. Then the concrete mixture is discharged from the mixing drum.

\subsection{DETERMINATION OF THE PARAMETERS OF THE MATHEMATICAL MODEL OF THE VIBRATION MIXER}

The main parameters influencing the choice of the mathematical model of the vibration mixer are the nature of the mixing drum vibrations, the coordinates of the center of gravity of the mixing drum, the physical and mechanical characteristics of the mixture, the geometric dimensions of the mixing drum and the volume of filling it with the concrete mixture.

When vibration in the vertical direction on the bottom of the mixing drum from the side of the processed concrete mixture, support forces will act, which can be represented in the form of inertial forces $F_{\text {in } 1}$ and dissipative forces $F_{d s 1}$, i.e. forces of inelastic resistance $F_{d s 1}$. These forces can be described by the following dependencies:

$$
\begin{aligned}
& F_{i n 1}=m_{p r 1} \frac{d^{2} z}{d t^{2}} ; \\
& F_{d s 1}=b_{p r 1} \frac{d z}{d t},
\end{aligned}
$$

where $m_{\rho r 1}$ - reduced mass of the concrete mixture interacting with the bottom of the mixing drum during vertically directed vibrations; $b_{p r 1}$ - reduced coefficient of inelastic resistance of the concrete mixture.

In this case, the value of the reduced mass of the concrete mixture can be determined from the dependence, modified as a result of the refinement, given in [6]:

$$
m_{\rho r 1}=\frac{\rho F_{1}}{k_{1}} \operatorname{tg} k_{1} H_{r}
$$

where $\rho$-dynamic density of the concrete mixture; $F_{1}$ - the area of the bottom of the mixing drum in contact with the concrete mixture; $k_{1}$ - the wavenumber; 


$$
k_{1}=\frac{\omega}{a_{1}}
$$

$a_{1}$ - phase speed of propagation of the disturbance in the treated layer:

$$
a_{1}=\sqrt{\frac{E}{\rho}} ;
$$

$E$ - dynamic modulus of elastic deformation of the processed concrete mixture; $H_{r}$ - estimated thickness of the processed layer, selected from the condition:

$$
\begin{aligned}
& \text { at } h \leq \frac{\pi}{4 \omega} \sqrt{\frac{E}{\rho}}, H_{r}=h ; \\
& \text { at } h>\frac{\pi}{4 \omega} \sqrt{\frac{E}{\rho}}, H_{r}=\frac{\pi}{4 \omega} \sqrt{\frac{E}{\rho}},
\end{aligned}
$$

where $h$ - thickness of the processed layer.

The coefficient of inelastic resistance of the concrete mixture is determined from the following relationship:

$$
b_{p r 1}=\eta_{1} k_{1} F_{1} \operatorname{tg} k_{1} H_{r}
$$

where $\eta_{1}$ - coefficient of dynamic viscosity of the concrete mixture;

$$
\eta_{1}=f_{v 1} H_{r} \sqrt{E \rho}
$$

where $f_{v 1}$ - coefficient of internal friction of the concrete mixture.

From the given dependences (4.2) and (4.8), let's find the specific values of the reduced mass and the coefficient of inelastic resistance:

$$
\begin{aligned}
& m_{y p r 1}=\frac{\rho}{k_{1}} \operatorname{tg} k_{1} m H_{r}, \\
& b_{y p r 1}=\eta_{1} k_{1} \operatorname{tg} k_{1} H_{r} .
\end{aligned}
$$

The specific values of the reduced mass and the coefficient of inelastic resistance of the concrete mixture interacting with the cylindrical walls of the mixing drum during horizontal vibrations are determined from the following dependencies:

$$
m_{y p r 2}=\frac{m_{y p r 1}}{2(1+\mu)}=\frac{\rho}{2 k_{1}(1+\mu)} \operatorname{tg} k_{1} L_{r}
$$




$$
b_{y p r 2}=\frac{b_{y p r 1}}{2(1+\mu)}=\frac{\eta_{1} k_{1}}{2(1+\mu)} \operatorname{tg} k_{1} L_{r}
$$

where $\mu$-Poisson's ratio; $L_{r}$ - length of the processed layer in the horizontal direction.

To determine the nature of the motion of the mixing drum in the vibration operating mode, let's consider the design diagram of the dynamic system «mixing drum - processed medium» (Fig. 4.2).
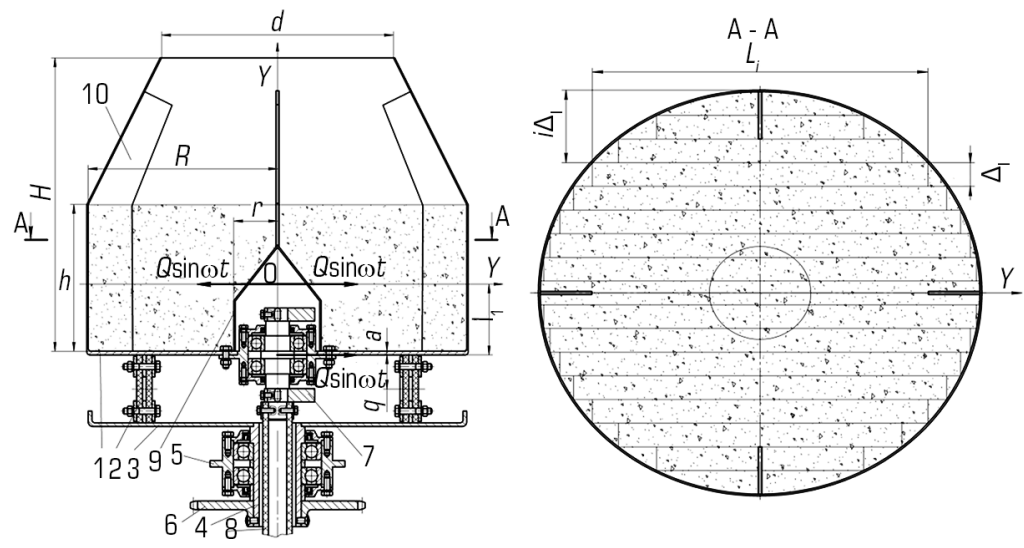

Fig. 4.2 Design diagram of the dynamic system «Mixing drum - processed medium»

To determine the value of the reduced mass and the coefficient of inelastic resistance of the concrete mixture interacting with the cylindrical walls of the mixing drum during horizontal vibrations in the direction of the coordinate, let's conventionally divide the entire mixed concrete mixture located in the cylindrical part of the mixing drum into a number of elementary vertical volumes. the same width (Fig. 4.2). From Fig. 4.2:

$$
\Delta_{l}=\frac{2 R}{n} \text {. }
$$

The length of each $i$-th volume with the width $\Delta_{l}$ located at a distance $i \Delta_{\text {, from the drum shell }}$ is determined from the following relationship:

$$
L_{i}=\sqrt{R^{2}+\left(R-i \Delta_{l}\right)^{2}} .
$$

Based on the obtained expressions (4.12), (4.13) and (4.15), let's determine the values of the reduced mass and the coefficient of inelastic resistance of the concrete mixture for each elementary volume interacting with the cylindrical walls of the mixing drum during horizontally directed vibrations: 


$$
\begin{aligned}
& m_{\text {ipr2 }}=\frac{h \Delta_{l} \rho}{2 k_{1}(1+\mu)} \operatorname{tg} k_{1} L_{i} ; \\
& b_{i p r 2}=\frac{h \Delta_{l} \eta_{1} k_{1}}{2(1+\mu)} \operatorname{tg} k_{1} L_{i} .
\end{aligned}
$$

\subsection{EQUATION OF MOTION AND ANALYTICAL DESCRIPTION OF VIBRATION MIXER MOTION}

Based on the obtained expressions (4.10), (4.11), (4.18) and (4.19) of the calculation scheme shown in Fig. $\mathbf{4 . 2}$ and the method described above, vibrations of a mixing drum loaded with concrete can be represented in the form of the following system of differential equations:

- vibrations in the horizontal direction along the coordinate axis:

$$
\left(m+m_{\rho r 2}\right) \frac{d^{2} y_{1 r}}{d t^{2}}+\left(b_{2}+b_{p r 2}\right) \frac{d y_{1 r}}{d t}+c_{2} y_{1 r}=Q \sin \omega t
$$

- angular (torsional) vibrations about the $X$ coordinate axis:

$$
\left(m+m_{\rho r 2}\right) \frac{d^{2} x_{1 r}}{d t^{2}}+\left(b_{3}+b_{p r 2}\right) \frac{d x_{1 r}}{d t}+c_{3} x_{1 r}=Q \cos \omega t ;
$$

- angular (torsional) vibrations about the $X$ coordinate axis:

$$
\left(J_{x}+J_{b 1}\right) \frac{d^{2} \psi_{x r}}{d t^{2}}+\left(n_{2}+n_{b 2}\right) \frac{d \psi_{x r}}{d t}+k_{2} \psi_{x r}=Q l_{1} \sin \omega t
$$

- angular (torsional) vibrations about the $Y$ coordinate axis:

$$
\left(J_{y}+J_{b 1}\right) \frac{d^{2} \psi_{y r}}{d t^{2}}+\left(n_{3}+n_{b 2}\right) \frac{d \psi_{y r}}{d t}+k_{3} \psi_{y r}=Q l_{1} \cos \omega t
$$

- vertical vibrations in the direction of the $Z$ coordinate axis along the $X$ axis:

$$
z_{x r}=x \psi_{y r}
$$

- vertical vibrations in the direction of the $Z$ coordinate axis along the $Y$ axis:

$$
z_{y r}=y \psi_{x r},
$$

where $x_{1 r}, y_{1 r}, y_{1 r}, z_{y r}$ - linear displacements of the mixing drum in the direction of the coordinate axes $X, Y$ and $Z$ under the action of harmonic exciting forces $Q \cos \omega t$ and $Q \sin \omega t$ in the operating mode; $\psi_{x r}, \psi_{y r}$ - angular displacements of the mixing drum relative to the coordinate 
axes $X, Y$, respectively, under the action of the moments of exciting forces $Q \sin \omega t$ and $Q \cos \omega t$ in the operating mode; $I_{1}$ - distance from the center of application of the exciting force of the vibration exciter of vibrations to the center of gravity of the vibration dynamic system in the operating mode in the direction of the coordinate $Z_{1}$,

$$
I_{1}=\frac{m l+m_{p r 1}(0.5 h+a)}{m+m_{p r 1}}
$$

$J_{b 1}-$ moment of inertia of the concrete mixture relative to the horizontal axis of gravity passing through the center of weight:

$$
J_{b 1}=\frac{1}{2} m_{p r 2} R^{2}+\frac{1}{12} m_{p r 2} h^{2}+m_{p r 2}\left(0.5 h+a-l_{1}\right)^{2}
$$

- coefficient of inelastic resistance of the concrete mixture at angular displacements of the oscillating system relative to the horizontal axis;

$$
n_{b 2}=0.5 h b_{p r 2} .
$$

The solution to the system of differential equations (4.18)-(4.21) for stationary oscillations describing the steady motion of a vertically installed mixing drum in operating mode is presented in the following form:

$$
\begin{aligned}
& y_{1 r}(t)=A_{1 r} \sin \left(\omega t-\varphi_{1 r}\right) \\
& x_{1 r}(t)=A_{2 r} \cos \left(\omega t+\varphi_{2 r}\right) ; \\
& \Psi_{x r}(t)=\Psi_{1 r} \sin \left(\omega t-\xi_{1 r}\right) \\
& \Psi_{y r}(t)=\Psi_{2 r} \cos \left(\omega t+\xi_{2 r}\right),
\end{aligned}
$$

where $A_{1 r}$ - amplitude of harmonic oscillations of the oscillating center of gravity of the system in the direction of the coordinate $Y$ in the operating mode; $A_{2 r}$-amplitude of harmonic oscillations of the center of gravity of the oscillating system in the direction of the coordinate $X$ in the operating mode; $\Psi_{1 r}$ - amplitude of angular (torsional) harmonic vibrations of the mixing drum relative to the axis $X$ passing through the center of gravity of the oscillating system in the operating mode; $\Psi_{2 r}$ - amplitude of angular (torsional) harmonic vibrations of the mixing drum relative to the axis $Y$ passing through the center of gravity of the oscillating system in the operating mode; $\varphi_{1 r}, \varphi_{2 r}$ - phase shift angle between the amplitudes of the exciting forces and the amplitudes of the forced vibrations; $\xi_{1 r}, \xi_{2 r}$ - phase shift angle between the amplitudes of the moments of the exciting forces and the amplitudes of the angular forced vibrations; 


$$
\begin{aligned}
& A_{1 r}=\frac{Q}{\sqrt{\left[c_{2}-\left(m+m_{p r 2}\right)\right]^{2}+\left(b_{2}+b_{p r 2}\right)^{2} \omega^{2}}} ; \\
& A_{2 r}=\frac{Q}{\sqrt{\left[c_{3}-\left(m+m_{p r 2}\right)\right]^{2}+\left(b_{2}+b_{p r 2}\right)^{2} \omega^{2}}} ; \\
& \Psi_{1 r}=\frac{Q l_{1}}{\sqrt{\left[k_{2}-\left(J_{x}+J_{b 1}\right)\right]^{2}+\left(n_{2}+b_{b 2}\right)^{2} \omega^{2}}} ; \\
& \Psi_{2 r}=\frac{\left.Q\right|_{1}}{\sqrt{\left[k_{3}-\left(J_{x}+J_{b 1}\right)\right]^{2}+\left(n_{3}+b_{b 2}\right)^{2} \omega^{2}}} ; \\
& \varphi_{1 r}=\operatorname{arctg} \frac{\left(b_{2}+b_{p r 2}\right) \omega}{c_{2}-\left(m+m_{p r 2}\right)} ; \\
& \varphi_{2 r}=\operatorname{arctg} \frac{\left(b_{3}+b_{p r 2}\right) \omega}{c_{3}-\left(m+m_{p r 2}\right)} \\
& \xi_{1 r}=\operatorname{arctg} \frac{\left(n_{2}+b_{b 2}\right) \omega}{k_{2}-\left(J_{x}+J_{b 1}\right)} ; \\
& \xi_{2 r}=\operatorname{arctg} \frac{\left(n_{3}+b_{b 2}\right) \omega}{k_{3}-\left(J_{x}+J_{b 1}\right)} .
\end{aligned}
$$

Using the obtained solutions (4.32)-(4.43) of the system of equations (4.18)-(4.28) of the considered dynamic system, let's successively determine the laws of motion of the bottom and walls of the mixing drum, as well as the protective cap, affecting the processed concrete mixture both in vertical and horizontal directions, and causing normal and tangential stresses in the processed medium, significantly affecting the destruction of internal bonds in the concrete mixture and, accordingly, the efficiency of vibration processing and mixing of the prepared concrete mixture.

The law of motion of the bottom of the mixing drum interacting with the processed concrete mixture horizontally in the direction of the coordinate can be described by the following equation:

$$
X_{d r}(t)=x_{1 r}(t)+(l-a) \psi_{y r}(t)=A_{2 r} \cos \left(\omega t+\varphi_{2 r}\right)+\Psi_{2 r}(l-a) \cos \left(\omega t+\xi_{2 r}\right),
$$

where $X_{d r}(t)$ - horizontal displacement of the mixing drum bottom in the direction of the coordinate $X$, which causes contacting stresses in the concrete medium.

After transformations of expression (4.44), let's obtain a dependence convenient for analysis and computer simulation:

$$
X_{d r}(t)=A_{2 d} \cos \left(\omega t+\theta_{2}\right)
$$


where $A_{2 d}$ - amplitude of harmonic vibrations of the bottom of the mixing drum in the direction of the coordinate axis,

$$
A_{2 d}=\sqrt{\left.A_{2 r}^{2}+\left[\Psi_{2 r}(l-a)\right]^{2}+2 A_{2 r} \Psi_{2 r}(l-a)\right] \cos \left(\varphi_{2 r}-\xi_{2 r}\right)}
$$

$\theta_{2}$ - angle of phase displacement between the amplitude of the exciting force and the amplitude of the forced vibrations of the bottom of the mixing drum in the direction of the coordinate axis $X$,

$$
\theta_{1}=\operatorname{arctg} \frac{A_{2 r} \sin \varphi_{2 r}+\Psi_{2 r}(l-a) \sin \xi_{2 r}}{A_{2 r} \cos \varphi_{2 r}+\Psi_{2 r}(l-a) \cos \xi_{2 r}}
$$

The law of motion of the bottom of the mixing drum interacting with the processed concrete mixture horizontally in the direction of the coordinate can be described by the following equation:

$$
Y_{d r}(t)=y_{1 r}(t)+(l-a) \psi_{x \Gamma}(t)=A_{1 r} \sin \left(\omega t-\varphi_{1 r}\right)+\Psi_{1 r}(l-a) \sin \left(\omega t-\xi_{1 r}\right),
$$

where $Y_{d r}(t)$ - horizontal displacement of the mixing drum bottom in the direction of the coordinate $Y$, which also causes contacting stresses in the concrete medium.

Transforming expression (4.48), let's obtain a dependence convenient for analysis and computer simulation:

$$
Y_{d r}(t)=A_{1 d} \sin \left(\omega t-\theta_{1}\right)
$$

where $A_{1 d}$ - amplitude of harmonic vibrations of the bottom of the mixing drum in the direction of the coordinate axis $Y$,

$$
A_{1 d}=\sqrt{\left.A_{1 r}^{2}+\left[\Psi_{1 r}(l-a)\right]^{2}+2 A_{1 r} \Psi_{1 r}(l-a)\right] \cos \left(\varphi_{1 r}-\xi_{1 r}\right)} ;
$$

$\theta_{1}$ - angle of phase displacement between the amplitude of the exciting force and the amplitude of the forced vibrations of the bottom of the mixing drum in the direction of the coordinate axis $X$,

$$
\theta_{1}=\operatorname{arctg} \frac{A_{1 r} \sin \varphi_{1 r}+\psi_{1 r}(\ell-a) \sin \xi_{1 r}}{A_{1 r} \cos \varphi_{1 r}+\psi_{1 r}(\ell-a) \cos \xi_{1 r}}
$$

The law of motion of the bottom of the mixing drum interacting with the processed concrete mixture in the vertical direction along the coordinate $Y$ can be described by the following equation:

$$
Z_{d r}(y, t)=y \Psi_{x r}(t)=\Psi_{1 r} y \sin \left(\omega t-\xi_{1 r}\right) \text { at }-R \leq y \leq-r, r \leq y \leq R .
$$


The law of motion of the bottom of the mixing drum interacting with the processed concrete mixture in the vertical direction along the coordinate $X$ can be described by the following equation:

$$
Z_{d r}(x, t)=x \psi_{y r}(t)=x \psi_{2 r} \cos \left(\omega t+\xi_{2 r}\right) \text { at }-R \leq x \leq-r, r \leq x \leq R .
$$

Here $Z_{d r}(y, t)$ and $Z_{d r}(x, t)$ - displacement of the bottom of the mixing drum in the vertical direction along the coordinates $X$ and $Y$, causing normal stresses in the concrete medium.

The law of motion of the mixing drum shell interacting with the processed concrete mixture horizontally in the coordinate $X$ direction can be described by the following equation:

$$
\begin{aligned}
& X_{o b r}(z, t)=X_{1 r}(t)+z \Psi_{y r}(t)= \\
& =A_{2 r} \cos \left(\omega t+\varphi_{2 r}\right)+\Psi_{2 r} z \cos \left(\omega t+\xi_{2 r}\right) \text { at }-(l-a) \leq z \leq(H-I+a),
\end{aligned}
$$

where $X_{\text {obr }}(z, t)$ - movement of the shell of the mixing drum in the horizontal direction along the coordinate $X$, which causes normal stresses in the medium being processed.

After transformations of expression (4.54), let's obtain a dependence convenient for analysis and computer simulation:

$$
X_{o b r}(z, t)=A_{3 o b}(z) \cos \left[\omega t+\theta_{3}(z)\right]
$$

where $A_{3 o b}$ - variable amplitude of harmonic vibrations of the mixing drum shell in the direction of the coordinate axis,

$$
A_{3 o b}=\sqrt{A_{2 r}^{2}+\left(\psi_{2 r} z\right)^{2}+2 A_{2 r} \psi_{2 r} z \cos \left(\varphi_{2 r}-\xi_{2 r}\right)} ;
$$

$\theta_{3}(z)$ - variable phase angle between the amplitude of the exciting force and the amplitude of forced vibrations of the shell of the mixing drum in the direction of the coordinate axis:

$$
\theta_{3}(z)=\operatorname{arctg} \frac{A_{2 r} \sin \varphi_{2 r}+\Psi_{2 r} z \sin \xi_{2 r}}{A_{2 r} \cos \varphi_{2 r}+\Psi_{2 r} z \cos \xi_{2 r}} .
$$

The law of motion of the mixing drum shell interacting with the processed concrete mixture horizontally in the coordinate direction can be described by the following equation:

$$
\begin{aligned}
& Y_{o b r}(z, t)=y_{1 r}(t)+z \Psi_{x r}(t)= \\
& =A_{1 r} \sin \left(\omega t-\varphi_{1 r}\right)+\Psi_{1 r} z \sin \left(\omega t-\xi_{1 r}\right) \text { at }-(I-a) \leq z \leq(H-I+a),
\end{aligned}
$$

where $Y_{\text {obr }}(z, t)$ - movement of the shell of the mixing drum in the horizontal direction along the coordinate, causing normal stresses in the medium being processed. 
Transforming expression (4.58), let's obtain a dependence convenient for analysis and computer simulation:

$$
Y_{o b r}(z, t)=A_{4 o b}(z) \sin \left[\omega t-\theta_{4}(z)\right]
$$

where $A_{40 b}$ - variable amplitude of harmonic vibrations of the mixing drum shell in the direction of the coordinate axis $Y$,

$$
A_{4 o b}=\sqrt{A_{1 r}^{2}+\left(\Psi_{1 r} z\right)^{2}+2 A_{1 r} \Psi_{1 r} z \cos \left(\varphi_{1 r}-\xi_{1 r}\right)}
$$

$\theta_{3}(z)$ - variable phase angle between the amplitude of the exciting force and the amplitude of forced vibrations of the shell of the mixing drum in the direction of the coordinate axis $Y$,

$$
\theta_{4}(z)=\operatorname{arctg} \frac{A_{1 r} \sin \varphi_{1 r}+\Psi_{1 r} z \sin \xi_{1 r}}{A_{1 r} \cos \varphi_{1 r}+\Psi_{1 r} z \cos \xi_{1 r}}
$$

The law of motion of the shell of the mixing drum interacting with the processed concrete mixture in the vertical direction along the coordinates $Y$ and $X$ can be described by the following equations:

- for the cylindrical part:

$$
\begin{aligned}
& Z_{\text {obyr }}(t)=R \psi_{x r}(t)=\Psi_{1 r} R \sin \left(\omega t-\xi_{1 r}\right) ; \\
& Z_{o b x r}(t)=R \psi_{y r}(t)=\Psi_{2 r} R \cos \left(\omega t+\xi_{2 r}\right)
\end{aligned}
$$

- for the tapered part:

$$
\begin{aligned}
& Z_{o b x r}(y, t)=R \Psi_{x r}(t)= \\
& =\Psi_{1 r}\left[R-\frac{y-(h-\ell-a)}{\operatorname{tg} \beta}\right] \sin \left(\omega t+\xi_{1 r}\right) \text { at }(h-\ell+a) \leq y \leq(H-\ell+a) ; \\
& Z_{o b x r}(x, t)=R \Psi_{y r}(t)= \\
& =\Psi_{2}\left[R-\frac{y-(h-l+a)}{\operatorname{tg} \beta}\right] \cos \left(\omega t+\xi_{2 r}\right) \text { at }(h-l+a) \leq y \leq(H-I+a),
\end{aligned}
$$

where $Z_{\text {obyr }}(t)$ and $Z_{\text {obxr }}(t)$ - displacement of the cylindrical part of the mixing drum in the vertical direction along the coordinates $Y$ and $X$, causing tangential stresses in the concrete medium; $Z_{o b y}(y, t)$ and $Z_{o b x}(x, t)$ - displacement of the tapered part of the mixing drum in the vertical direction along the coordinates $Y$ and $X$. 


\subsection{DETERMINATION OF THE MAIN PARAMETERS OF THE WORKING PROCESS OF AN OPERATION OF CONCRETE MIXER}

Based on the obtained theoretical dependencies (4.1)-(4.65), describing the vibrations of the vibration drum in the operating mode, a program was created in the TurboPascal system designed to simulate the laws of motion of the mixing drum under the action of disturbing forces generated by the displaced vibration exciter of circular vibrations.

Fig. 4.3-4.6 show the theoretical values of the vibration amplitudes of the surfaces of a vibration drum in contact with a concrete mixture of different consistency in the horizontal plane and in the vertical direction. Used cement-concrete mixtures with a cone subsidence $O K=3.5-4 \mathrm{~cm}$ and a stiffness of $30 \mathrm{~s}, 60 \mathrm{~s}$ and $90 \mathrm{~s}$.

The data of the vibration amplitudes of the bottom of the mixing drum (Fig. 4.3, a) and the shell of the mixer drum (Fig. $\mathbf{4 . 3}, \boldsymbol{b}$ ) with horizontal (1) and vertical (2) movements in the operating mode are given. The calculations were performed for the preparation of a concrete mixture with a cone subsidence $O K=3.5-4 \mathrm{~cm}$.
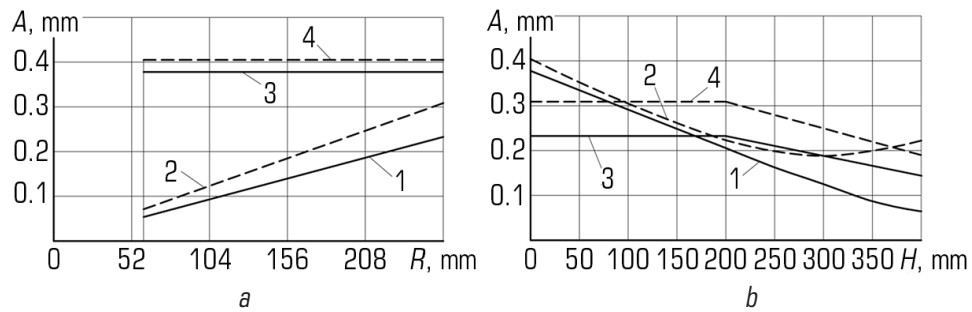

O Fig. 4.3 Amplitudes of vibrations of the surfaces of the mixing drum (mixture with cone subsidence $O K=3.5-4 \mathrm{~cm}$ )
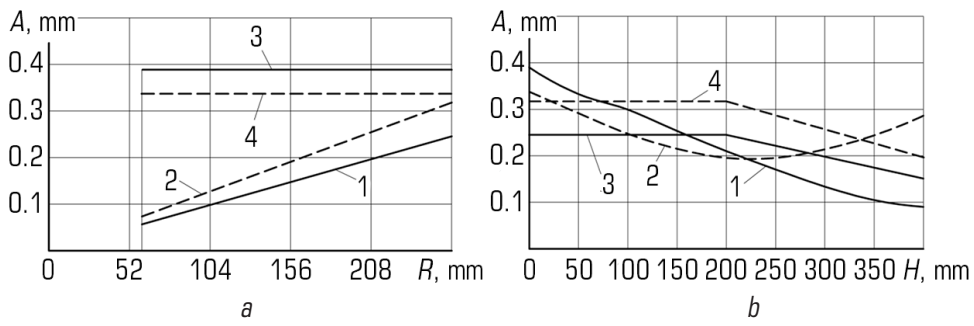

O Fig. 4.4 Amplitudes of vibrations of the surfaces of the mixing drum (mixture with a hardness of $30 \mathrm{~s}$ ) 

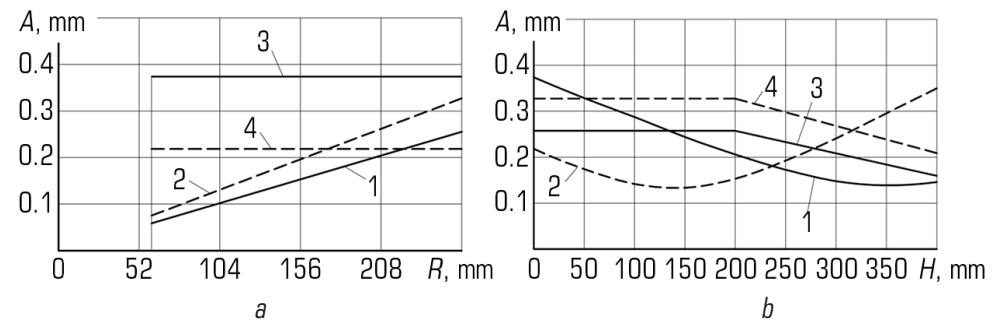

- Fig. 4.5 Amplitudes of vibrations of the surfaces of the mixing drum (mixture with a hardness of $60 \mathrm{~s}$ )
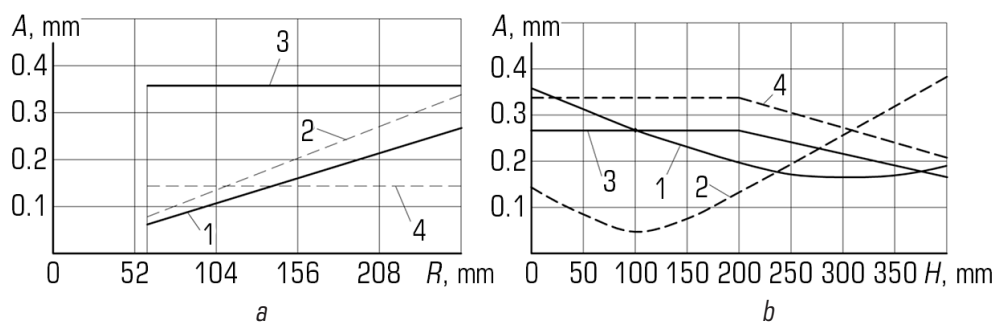

O Fig. 4.6 Amplitudes of vibrations of the surfaces of the mixing drum (mixture with a hardness of $90 \mathrm{~s}$ )

When preparing hard concrete mixes, the distribution of amplitudes is somewhat different from mobile mixes. Yes, in $\mathbf{F i g}$. 4.5, 4.6 show the results of calculations for mixtures with stiffness of $60 \mathrm{~s}$ and $90 \mathrm{~s}$ for the bottom of the mixer drum (a) with horizontal (1) and vertical (2) and the shell of the mixer drum (b) with horizontal (1) and vertical (2) movements.

The data presented show that during the preparation process concrete mixtures are subjected to vibration action with sufficiently high amplitudes in the horizontal plane and in the vertical direction at a frequency of angular vibrations of $292 \mathrm{rad} / \mathrm{s}$, sufficient for effective mixing and vibration activation of the concrete mixture.

Thus, the obtained expressions (4.1)-(4.65) make it possible to establish the law of motion of all surfaces of the inner surface of the mixer interacting with the processed cementconcrete mixture during its preparation, to determine the main parameters of the proposed vibration device depending on the physical and mechanical characteristics of the processed mixture, to justify rational modes. actions on the concrete mix during its vibration processing and preparation.

For experimental research, a vibration mixer was developed and manufactured (Fig. 4.7). 


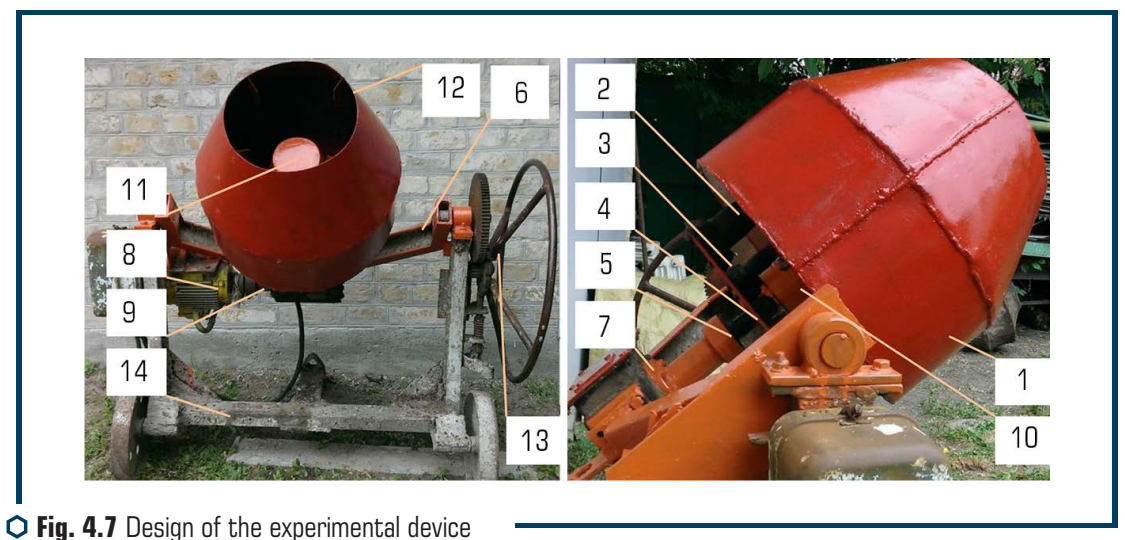

It is a cone-cylindrical mixing drum 1, which is mounted on a base plate 3 with the help of elastic shock absorbers 2 . This base plate 3 is rigidly connected in its central part to a drive shaft 4 installed in a bearing support 5 . A worm is built into the cross-beam 6 a gearbox 7 connected to an electric motor 8 through a clutch 9 . On the bottom of the mixing drum 1, a circular vibration exciter 10 is mounted, which is half mounted in the inner cavity of the mixing drum. Inside the mixing drum, the circular vibration exciter 10 is closed with a conical protective cap 11, which is hermetically attached to the bottom of the drum. Inside the mixing drum 1 there are blades 12 . The traverse 6 and the locking and tipping mechanism 13 are mounted on the frame 14.

The process of testing the operation of the mixer with the necessary instruments for measuring parameters was carried out in accordance with the requirements of the interstate standard (GOST 16349-85).

- error of power measuring instruments - no more than $2.5 \%$;

- rotational speed of the mixing bodies was checked under load;

- weight of the mixer was determined by weighing on a balance;

- total time of one mixing cycle was determined by a stopwatch and was counted from the beginning of loading the mixture to the end of unloading the mixed mixture;

- composition, grade of the concrete mixture was determined at the test site;

- during the tests, three batches of mixtures of the same composition were prepared. The amount of the mixture was sufficient for testing. A total of three mixtures were used.

All indicators provided by the interstate standard, including vibration parameters (amplitude and frequency of oscillations), were recorded through the appropriate equipment on the computer display (Fig. 4.8).

On the foundation on the side of the vibration mixer (Fig. 4.8) there is a fixed stand of the tripod, in which the sensor is fixed on a special clamp. The other end of the sensor is attached to the bottom of the mixing drum through a special bracket. The length of the sensor base and 
its thickness are selected from the condition that the natural oscillation frequency of the sensor $\omega_{0 d}$ exceeds the value of the oscillation frequency by $8 . . .10$ times.

As a result of the experiments, the value of the vibration amplitude is recorded on the computer screen in the form of vibrograms. Based on the results of the analysis and processing of vibration records, both the mutual influence of the working body and the medium and the determination of optimal modes are established. The analysis of the records was carried out in two stages: preliminary analysis of the oscillogram; complete control processing.

By processing a series of typical vibrograms according to the above method, the numerical values of the vibration amplitudes of the bottom of the mixing drum and shell were obtained for mixtures with different cone draft and different stiffness. Based on the results of data processing, graphs were constructed with the repetition of each experiment in three dimensions. Fig. $\mathbf{4 . 9}$ shows graphs comparing the theoretical and experimental values of the amplitude of oscillations of horizontal and vertical oscillations of the bottom and shell, depending on the radius, measured from the axis of rotation of the drum to the shell of the drum.

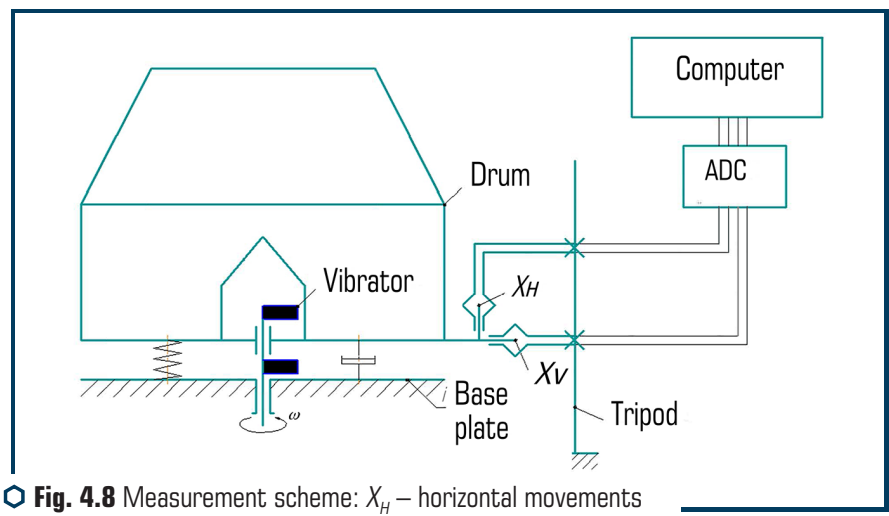

of the mixing drum; $X_{V}$ - vertical movements of the mixing drum

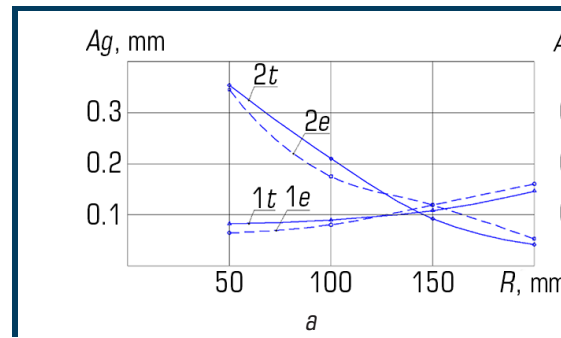

$A b, \mathrm{~mm}$

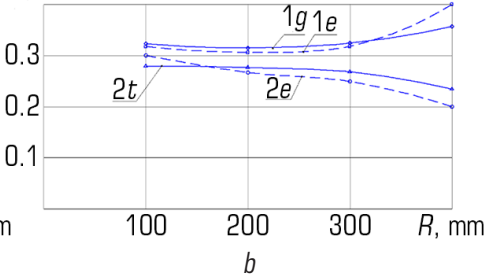

O Fig. 4.9 Comparison of theoretical and experimental values of the amplitude:

$a$ - horizontal vibrations of the bottom and shell, depending on the radius;

$b$ - vertical vibrations of the bottom and shell for the mixture $G=30 \mathrm{~s}$ 
An assessment of the performed studies has established that the discrepancy between the theoretical and experimental numerical values in the vibration amplitudes is $11-15 \%$.

\subsection{METHODOLOGY AND ALGORITHM FOR CALCULATING THE MAIN PARAMETERS OF A VIBRATION CONCRETE MIXER}

The initial parameters are the amplitude, the vibration frequency, set depending on the composition of the mixture. An important indicator of the vibration process is the selected type and direction of vibrations and the nature of vibrations. If, according to the condition of the problem, the values of the amplitudes and frequencies of oscillations are absent, then their values can be determined based on the fact that the dominant parameter is acceleration. Then such properties as amplitude, speed, frequency, acceleration must be considered together.

For harmonic oscillations with frequency, the oscillation amplitude is optimal [7]:

$$
X_{\text {OPT }}=\frac{(4-6) g}{\omega^{2}} \text {, }
$$

where $g$ - acceleration due to gravity $(g=9.8 \mathrm{~m} / \mathrm{s})$. For the frequency $\omega=3.14 \mathrm{~s}^{-1}$, the optimal vibration amplitude is determined by the lower and upper acceleration, i.e. $X_{\text {OPT }}=0.4-0.6 \mathrm{~mm}$.

The second parameter is the drive power of the concrete mixer, which is spent on vibrations of the mixture $P_{v}$ and friction in the bearings of the vibration exciter $P_{F R}$.

The vibration power is calculated according to the maximum value that the existing driving force can develop with an increase in the dissipative resistance of the system.

Power per vibration, $W$, is determined by the dependence [7]:

$$
\max P_{a v}=\frac{1}{4} F_{0} x_{c}^{\prime} \omega
$$

where $F_{0}$ - driving force necessary to maintain a given amplitude is calculated taking into account the effect of the concrete mixture on the working body of the machine; $x_{c}^{\prime}$ - amplitude of displacements of the working body, taking into account only the reactive forces of the system:

$$
x_{c}^{\prime}=\frac{m_{0} r_{0}}{m_{c} a+m}, m_{c}=\frac{\rho F}{k} \operatorname{tg} k H,
$$

where $\omega$ - frequency of forced vibrations.

Friction force power in vibration exciter bearings, W,

$$
P_{F R}=F_{0} \mu \frac{d_{j}}{2} \omega \text {, }
$$

where $\mu=0.005 \ldots . .0 .008$ - coefficient of friction in the bearings; $d_{j}$ - diameter of the bearing journal. 
Motor power for vibration exciter, kW:

$$
P_{M}=\frac{\max P_{A V}+P_{F R}}{1000 \eta}
$$

where $\mu$-transmission efficiency.

The main parameters of the gravitational action, as components of the general process of mixing the concrete mixture, is the drum rotation frequency, which is determined by the dependence:

$$
\omega=\sqrt{2 g / D} .
$$

The algorithm for calculating the main parameters and characteristics of a vibration mixer based on gravitational and vibration components is shown in Fig. 4.10.

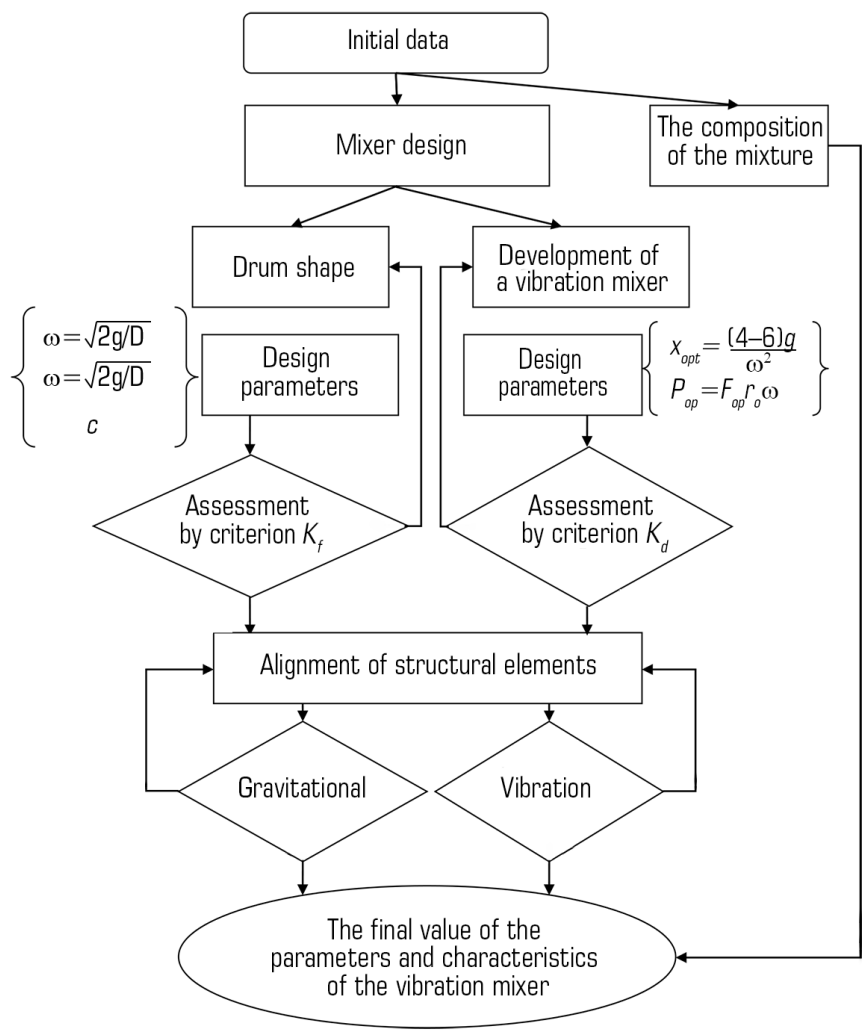

Fig. 4.10 Algorithm for calculating the parameters and characteristics of a vibration mixer 


\subsection{DISCUSSION OF RESEARCH RESULTS}

The analysis of the obtained expressions (4.32)-(4.65) shows that the mixing drum in the process of operation makes complex spatial vibrations, designed to provide an effective vibration action on the mixed concrete mixture. Moreover, each point located on the inner surface of the mixing drum has its own law of motion. As a result, the mixed concrete mixture will experience an alternating amplitude-frequency action, which causes multidirectional normal and tangential stresses in the mixture, which ensures the ultimate destruction of internal bonds and greater mobility in it, which contributes to an increase in mass transfer and effective mixing [8-13].

The use of a vibration concrete mixer makes it possible to reduce the mixing time by 2.5-3 times in comparison with conventional free mixing concrete mixers. Provides the preparation of hard concrete mixes and improving the quality of concrete mixes due to the destruction of defective aggregates and a more uniform distribution of the binder throughout the volume of the mixed mix. An experimental design of vibration-gravitational mixing has been developed and manufactured, and studies of the working process of mixing efficiency have been carried out on it. Research carried out at three warehouses of concrete mixtures confirmed the assumptions accepted in the work, the assumption and the selected physical and mathematical model of the "Mixer - concrete mixture» system. It was found that the use of vibration and gravitational action makes it possible to reduce the mixing time from 90 to $60 \mathrm{~s}$. The strength of control concrete cubes is 17-18\% higher than in a gravity mixer. The discrepancy between the theoretical and experimental numerical values for the vibration amplitudes is 11-15\%. The proposed design and the studies carried out on it have confirmed the effectiveness of the vibration mixing method. As a result of the studies performed, it was found that the nature of the vibration of the mixing drum is close to harmonious [14]. The amplitude of vertical oscillations of the bottom varies in the range of $0.3-0.4 \mathrm{~mm}$ at a distance $R=208-220 \mathrm{~mm}$ from the drum bottom. At a distance of $R=120 \mathrm{~mm}$, the vibration amplitude slightly decreased and its average value is $0.2 \mathrm{~mm}$. Horizontal displacements of the drum bottom have huge vibration amplitudes $0.02 \mathrm{~mm}$. When loading with a concrete mixture, the amplitudes of vibrations at the same radius have significantly decreased, and their average value fluctuates between $0.2-0.25 \mathrm{~mm}$ and $0.14-0.16 \mathrm{~mm}$.

\section{CONCLUSIONS TO SECTION 4}

1. Well-coordinated equations of motion of the system «mixer - concrete mixture» and their solutions established the regularities of changing the parameters of the mixer at different angles of inclination.

2. The distribution of the amplitudes of vibrations of the working body of the vibration solvent has been obtained, depending on the distance of application of the energy source.

3. An experimental design of vibration-gravitational mixing has been developed and manufactured, and studies of the working process of mixing efficiency have been carried out on it. 
4. It has been determined that the use of a vibration concrete mixer makes it possible to reduce the duration of mixing by 2.5-3 times in comparison with conventional free mixing concrete mixers. Provides the preparation of hard concrete mixes and improving the quality of concrete mixes due to the destruction of defective aggregates and a more uniform distribution of the binder throughout the volume of the mixed mix.

5. The results of experimental studies are presented and a comparative analysis based on theoretical data has been carried out.

It has been found that the use of vibration and gravitational action can reduce the mixing time from 90 to $60 \mathrm{~s}$. The strength of control concrete cubes is 17-18\% higher than in a gravity mixer.

6. An algorithm and method for calculating the main parameters of a vibration concrete mixer have been developed.

\section{REFERENCES}

1. Nazarenko, I. I. (1999). Mashyny dlia vyrobnytstva budivelnykh materialiv. Kyiv: KNUBA, 488.

2. Nazarenko, I. I., Tumanska, O. V. (2004). Mashyny i ustatkuvannia pidpryiemstv budivelnykh materialiv. Konstruktsii ta osnovy ekspluatatsii. Kyiv: Vyshcha shkola, 590.

3. Emelianova, I. A., Dobrokhodova, O. V., Anischenko, A. I. (2010). Sovremennye stroitelnye smesi i oborudovanie dlia ikh prigotovleniia. Kharkiv: Timchenko, 146.

4. Bogomolov, A. A. (2010). Teoreticheskie i tekhnicheskie osnovy sovershenstvovaniia smesitelnykh mashin dlia prigotovleniia stroitelnykh smesei. Belgorod: Iz-vo BGTU, 151.

5. Ruchynskyi, M. M., Svyrydiuk, D. Ya. (2013). Doslidzhennia kolyvan vibratsiinoho betonozmishuvacha z urakhuvanniam vplyvu peremishchuvanoho materialu. Tekhnika budivnytstva. Naukovo-tekhnichnyi zhurnal, 31, 35-42.

6. Maslov, A. G., Ponomar, V. M. (1985). Vibratsionnye mashiny i protsessy v dorozhnom stroitelstve. Kyiv: Budivelnik, 128.

7. Nazarenko, I. I. (2007). Vibratsiini mashyny i protsesy budivelnoi industrii. Kyiv: KNUBA, 230.

8. Bernyk, I., Luhovskyi, O., Nazarenko, I. (2018). Effect of rheological properties of materials on their treatment with ultrasonic cavitation. Materiali in Tehnologije, 52 (4), 465-468. doi: http://doi.org/10.17222/mit.2017.021

9. Nazarenko, I. I., Ruchynskyi, M. M., Sviderskyi, A. T., Kobylanska, I. M., Harasim, D., Kalizhanova, A., Kozbakova, A. (2019). Development of energy-efficient vibration machines for the buiding-and-contruction industry. Przeglad Elektrotechniczny, 95 (4), 53-59. doi: http://doi.org/ 10.15199/48.2019.04.10

10. Nazarenko, I., Dedov, O., Bernyk, I., Rogovskii, I., Bondarenko, A., Zapryvoda, A. et. al. (2020). Determining the regions of stability in the motion regimes and parameters of vibratory machines for different technological purposes. Eastern-European Journal of Enterprise Technologies, 6 (7 (108)), 71-79. doi: http://doi.org/10.15587/1729-4061.2020.217747 
11. Nesterenko, M., Nazarenko, I., Molchanov, P. (2018). Cassette Installation with Active Working Body in the Separating Partition. International Journal of Engineering \& Technology, 7 (3.2), 265-268. doi: http://doi.org/10.14419/ijet.v7i3.2.14417

12. Nazarenko, I., Gaidaichuk, V., Dedov, O., Diachenko, 0. (2018). Determination of stresses and strains in the shaping structure under spatial load. Eastern-European Journal of Enterprise Technologies, 6 (7 (96)), 13-18. doi: http://doi.org/10.15587/1729-4061.2018.147195

13. Nazarenko, I., Svidersky, A., Kostenyuk, A., Dedov, O., Kyzminec, N., Slipetskyi, V. (2020). Determination of the workflow of energy-saving vibration unit with polyphase spectrum of vibrations. Eastern-European Journal of Enterprise Technologies, 1 (7 (103)), 43-49. doi: http:// doi.org/10.15587/1729-4061.0.184632

14. Nazarenko, I., Gavryukov, O., Klyon, A., Ruchynsky, N. (2018). Determination of the optimal parameters of a tubular belt conveyor depending on such an economical. Eastern-European Journal of Enterprise Technologies, 3 (1 (93)), 34-42. doi: http://doi.org/10.15587/17294061.2018.131552 